\title{
Pleuropulmonary Amoebiasis: Know to Think About
}

A Aissa ${ }^{1}$, M Hachicha ${ }^{1}$, A Daadoucha ${ }^{1}$, I Ben Othmen ${ }^{1}$, S Aissa ${ }^{2}$, H Barhoumi $^{3}$, M Benzarti $^{2}$ and R Alouini ${ }^{1}$

${ }^{1}$ Departement of Imaging, Ibn El Jazzar Hospital, Kairouan, Tunisia

${ }^{2}$ Departement of Chest Diseases, Farhat Hached Hospital, Sousse, Tunisia

${ }^{3}$ Department of Anesthesia, Surgical Unit Aghlabites, Kairouan, Tunisia

\begin{abstract}
Amoebiasis is the third leading cause of mortality due to parasitic infection worldwide after malaria and schistosomiasis. The direct pulmonary Entamoeba histolytica is rarer than reactive events called "neighborhood". We report the role of imaging in the diagnostic orientation in a case of amoebic pleuropulmonary damage (associated with serology and parasitology). Before an excavated pulmonary lesion, imaging can be a great contribution in the working diagnosis of pulmonary parasite Entamoeba histolytica.
\end{abstract}

Keywords: Amoebiasis; Lung imaging

\section{Introduction}

Amoebiasis is the third leading cause of death due to a parasitic infection in the world after malaria and schistosomiasis [1]. The pleuropulmonary localization is the second most common extra-intestinal expression after liver damage [1]. The direct pulmonary Entamoeba histolytica infestation is less common than secondary form called "neighborhood" reaction [2]. The authors propose to report a case of amoebic pleuropulmonary disease and highlight the contribution of imaging for the diagnosis orientation.

\section{Case Report}

A 56-year-old diabetic patient was admitted to intensive care unit for impaired general condition, oscillating fever, dry cough and weight loss. These symptoms were not resolved by antibiotics which aimed for community-acquired pneumonia. The evolution was marked by a worsening with persistent fever and installation of a brownish vomiting. The chest X-rays showed a diffuse alveolar filling associated with bilateral excavated images which the largest was right apical. A Complementary CT revealed bilateral and asymmetrical alveolar syndrome with parenchymal condensation some of which are confluent, containing multiple excavated images (Figures 1 and 2). There was a draining bronchus issued from the right lower lobe which was better visualized on MinIP reconstruction (Figure 3).

Bronchoscopy associated with parasitological test revealed the

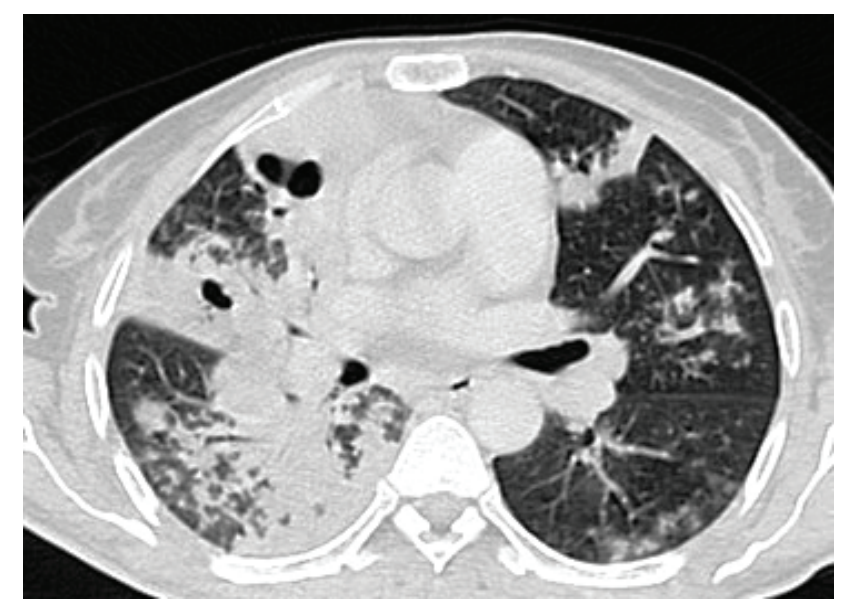

Figure 1: Chest CT: Axial plan in lung window. Right pulmonary parenchymal condensation with multiple excavated lesions.
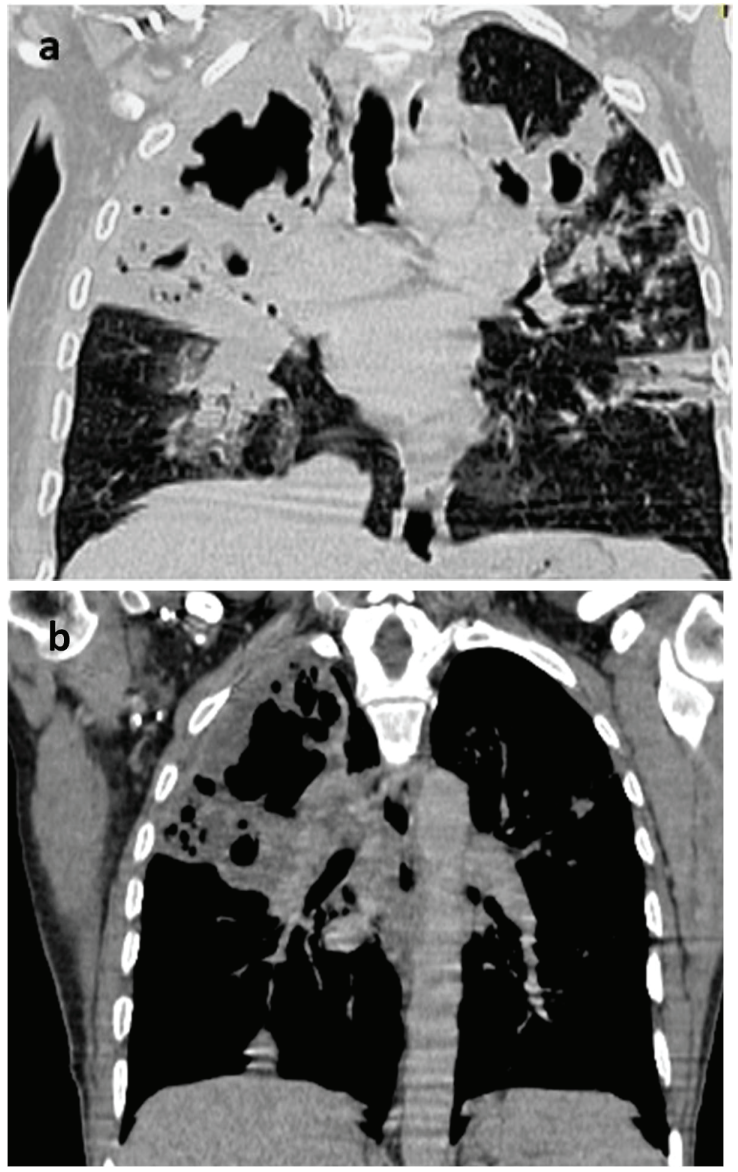

Figure 2: CT of the chest. Coronal reconstruction after injection of iodinated contrast in parenchymal window (a) and mediastinal (b). Bilateral alveolar filling Homes more pronounced on the right seat of parenchymatous condensation with a wide central excavation.

*Corresponding author: AAissa, Departement of Imaging, Ibn El Jazzar Hospital, Kairouan, Tunisia, Tel: 0021653848829; E-mail: amene.aissa@rns.tn

Received October 21, 2016; Accepted May 29, 2017; Published May 31, 2017

Citation: Aissa A, Hachicha M, Daadoucha A, Ben Othmen I, Aissa S, et al. (2017) Pleuropulmonary Amoebiasis: Know to Think About. J Pulm Respir Med 7: 409. doi: 10.4172/2161-105X.1000409

Copyright: ( 2017 Aissa A, et al. This is an open-access article distributed under the terms of the Creative Commons Attribution License, which permits unrestricted use, distribution, and reproduction in any medium, provided the original author and source are credited. 


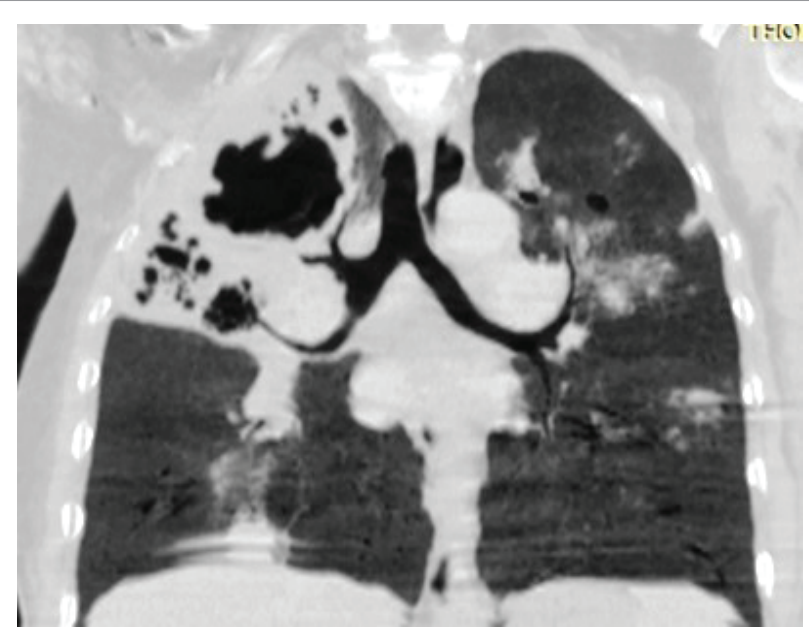

Figure 3: CT of the chest. Coronal reconstruction reconstruction MinIP. Demonstration of a bronchus of drainage from the right lower lobe communicating with the excavation the bronchial tree.

presence of the parasite Entamoeba histolytica. Amoebic serology was positive. Abdominal ultrasonography did not find any liver damage. The diagnosis of primary amoebic lung disease was made.

\section{Discussion}

Amoebiasis is a cosmopolitan disease; it prevails especially in hot countries of the Third World. This is the third cause of parasitelinked-death in the world after malaria and schistosomiasis [3]. It is currently estimated that $12 \%$ of the world population is affected by this germ among which only $10 \%$ are symptomatic. This is due to the fact that there are two strains of pathogenic and non-pathogenic amoebae and the majority of subjects are infested with non-pathogenic form [2]. In our country, both intestinal and visceral amoebiasis are relatively rare, the prevalence of intestinal parasitism by Entamoeba histolytica and Entamoeba dispar has been estimated between $0.85 \%$ and $1.86 \%$. However, the visceral localization was rarely reported [4] especially pleuro-pulmonary lesions which are the rarest extraintestinal expression (after liver disease) [1-3,5,6]. Pleuro-pulmonary involvement occurs almost exclusively in patients with liver abscess. This "secondary" form is spread through intra-thoracic way which is preferably trans- phrenic, whether by "continuity" by symphysis between liver - diaphragm - pleura and lung base or "contiguity" by progress through the per hepatic, peritoneal, pleural and pulmonary lymphatic anastomoses. This Trans phrenic route is the most common and explains the predominance of right chest locations. More rarely, the break of the hepatic veins allows access for the amoeba to the right heart then to the lung. This path, called "primitive" or "vascular", may explain multifocal amoebic, atypical and without preexisting liver disease forms [2-5]. In our case, the absence of hepatic localization suggests either a heterogeneous transmission or a migration by contiguity from an unnoticed sub-diaphragmatic abscess. Moreover, it is sometimes impossible to diagnose a sub-diaphragmatic abscess other than by necropsy [2]. The pathogenesis of lung localization itself is now well known, although it should be clearly distinguished between direct lung localization and secondary forms, which prognosis, depending on a timely diagnosis, can be quite different $[2,6]$. Although direct pulmonary infestation is too rare, it requires an early diagnosis under penalty of sequelae. Clinically, an uncomplicated lung amoebic disease differs little from an ordinary pneumonia. However, ignoring amoebic lung disease or treating it as a community-acquired pneumonia may lead to a potentially severe condition $[1,3,5]$. Therefore, the clinician must rely, in addition to clinical and epidemiological data, mainly on radiological, parasitological and serological data that will guide the diagnosis. Chest radiographs remain the first-line radiological test. It can show a non or systematic opacity of the right lung, a stripcondensation of the right base, or seldom "balloon release" images or atypical locations (right upper lobe, left lung) related to hematogenous spread $[2,6]$. The presence of an excavated or hydro-aeric image within a pulmonary infiltrate is also in favor of the diagnosis. In our case, the front chest radiograph showed bilateral excavated images which the most voluminous was right; this bilateral aspect is consistent with the primitive or vascular dissemination mode. The "excavated" appearance of lesions may explain the observed chocolate colored-vomiting in our patient. Chest CT showed, on its side, a symmetrical bilateral alveolar syndrome containing excavated images which is in favor of amoebic infection. In total, amoebic etiology should be considered in preferential right localization, oscillating and persisting fever despite classical antibiotics and especially the traditional "vomiting " of "pus chocolate", which is odorless and germ-free in microbial culture $[1,2]$.

\section{Conclusion}

The pleuro-pulmonary amoebiasis can be a complication of invasive amoebic infestations, especially in endemic areas. Given its rarity and for an early diagnosis, imaging next to parasitological examination and serology, plays a very important role in the diagnostic strategy especially in front of a right basal pneumonia with a trailing evolution.

\section{References}

1. Faye PM, Diagne-Gueye NR, Ba ID, Fall AL (2011) Pulmonary amoebiasis in children. J Pedia Child Care $24: 284-287$.

2. Dubut F, Benhamou D, kouziaeff N (2000) Muir: Respiratory infection with Entamoeba histolytica. Rev Mal Respir 17: 878-880.

3. Rachid H, Aloui YA, loudadssi F, El Biaze M (2005) Pleuropulmonary lesions at Entamoeba histolytica. Rev Mal Respir 22: 1035-1037.

4. Makni F, Sellami H, Sellami A, CHeikheouhou F, Ayadi H (2007) The visceral amoebiasis: Assessment in the CHU of Sfax. Rev Tun Infectiol 1: 20-24.

5. Shenoy VP, Vishwanath S, Indira B, Rodrigues G (2010) Hepatopulmonary amebiasis: A case report. Braz J Infect Dis 14: 372-373.

6. Ayadi H, Rekik WK, Ayoub K (2001) Bilateral excavated nodular pneumonia: Amoeba responsible? Rev Pneumol Clin 57: 4347. 